\title{
Supplementation With Highly Purified Docosahexaenoic Acid Ethyl Ester Was Associated With Enhancement of Hepatic Fatty Acid Oxidation and Greater Apparent Absorption of Docosahexaenoic Acid in Rats
}

\author{
Kenji Fukunaga ${ }^{1}$, Ryota Hosomi ${ }^{2}$, Ryosuke Inayoshi ${ }^{1}$, Toshimasa Nishiyama ${ }^{3} \&$ Munehiro Yoshida $^{1}$ \\ ${ }^{1}$ Department of Life Science and Biotechnology, Faculty of Chemistry, Materials and Bioengineering, Kansai \\ University, Osaka, Japan \\ ${ }^{2}$ Division of Human Living Sciences, Tottori College, Tottori, Japan \\ ${ }^{3}$ Department of Public Health, Kansai Medical University, Osaka, Japan \\ Correspondence: Kenji Fukunaga, Department of Life Science and Biotechnology, Faculty of Chemistry, \\ Materials, and Bioengineering, Kansai University, 3-3-35 Yamate-cho, Suita, Osaka 564-8680, Japan. Tel: \\ 81-663-68-0875. E-mail: fukunagk@kansai-u.ac.jp
}

Received: June 4, 2013 Accepted: August 10, 2013 Online Published: August 26, 2013

doi:10.5539/jfr.v2n5p90 URL: http://dx.doi.org/10.5539/jfr.v2n5p90

\begin{abstract}
Fish oil-derived fatty acid ethyl ester (EE) has been widely utilized as a supplement in Europe and United States. However, few studies have assessed the apparent absorption of docosahexaenoic acid (DHA) between non-purified DHA-EE (L-DHAEE) and highly purified DHA-EE (H-DHAEE). This study evaluated the effects of L-DHAEE and H-DHAEE on lipid metabolism and apparent absorption of DHA in rats. Groups of male Wistar rats were fed AIN93G diet containing 2.8\% L-DHAEE or 1.0\% H-DHAEE for 4 or 8 weeks. As parameters of lipid metabolism and apparent absorption of DHA-EE, serum and liver lipid contents, fecal EE excretion, lipid-metabolizing enzyme activities, and organ fatty acid composition were measured. The L-DHAEE diet increased fecal DHA-EE and total EE excretion compared with the H-DHAEE diet. In rats fed DHA-EE for 4 weeks, H-DHAEE was more efficacious than L-DHAEE at increasing liver DHA content, partly owing to the enhancement of apparent absorption. In addition, rats fed the H-DHAEE diet had higher fatty acid $\beta$-oxidation activity in peroxisomes than rats fed the L-DHAEE diet for 8 weeks. These results suggest that H-DHAEE supplementation may have beneficial functions in the prevention of lifestyle-related diseases as compared with L-DHAEE supplementation.
\end{abstract}

Keywords: docosahexaenoic acid, fatty acid ethyl ester, apparent absorption, lipid metabolism

List of abbreviations: A/G, albumin/globulin; ACC, acetyl-CoA carboxylase; ACOX, Acyl-CoA oxidase; ALT, alanine aminotransferase; ANOVA, analysis of variance; AST, aspartate aminotransferase, BW, body weight; CES-II, carboxyl esterase-II; CPT-II, carnitine palmitoyl transferase-II; DHA, docosahexaenoic acid; EE, ethyl ester; EPA, eicosapentaenoic acid; FAS, fatty acid synthase; G6PDH, glucose-6-phosphate dehydrogenase; GAPDH, glyceraldehyde 3-phosphate dehydrogenase; GLC, gas-liquid chromatography; H-DHAEE, highly purified docosahexaenoic acid-ethyl ester; H-EE, highly purified docosahexaenoic acid-ethyl ester-containing diet; H-EE1.0, 1.0\% highly purified docosahexaenoic acid-ethyl ester-containing diet; L-DHAEE, non-purified docosahexaenoic acid-ethyl ester; L-EE, non-purified docosahexaenoic acid-ethyl ester-containing diet; L-EE1.0, $1.0 \%$ non-purified docosahexaenoic acid-ethyl ester-containing diet; L-EE2.8, 2.8\% non-purified docosahexaenoic acid-ethyl ester-containing diet; ME, malic enzyme; N.D., not determined; PL, phospholipid; PUFA, polyunsaturated fatty acid; SEM, standard error of the mean; SUN, serum urea nitrogen; TG, triacylglycerol; WAT, white adipose tissue.

\section{Introduction}

n-3 polyunsaturated fatty acids (PUFAs), such as and eicosapentaenoic acid (EPA) and docosahexaenoic acid (DHA), contained in fish oil have health-promoting effects, including anti-thrombotic, anti-atherogenic, and 
anti-hyperlipidemia effects (Hosomi et al., 2012). Based on their health-promoting effects, the intake of EPA and DHA has been recommended by national and international societies (Kris-Ethetron et al., 2003; Kris-Ethetron et al., 2002). EPA and DHA are sold as supplements contained in purified fish oil (triacylglycerol (TG)-form, phospholipid (PL)-form) or fish oil-derived fatty acid ethyl ester (EE). On the market, TG-form and EE-form are mainly supplied as supplements with fish oil. The advantage of the TG-form containing EPA and DHA is that it is a natural component of the human diet; however, with the TG-form it is difficult to concentrate specific fatty acids, such as EPA and DHA. Meanwhile, with the EE-form it is easy to obtain highly purified levels of specific fatty acids, but the EE-form is not a natural product. Moreover, there are some questions regarding the absorbability, effectiveness, and safety of the EE-form. The EE-forms of EPA and DHA showed lower intestinal absorption than the TG-forms and the free acid forms (Ikeda et al., 1993). The low absorbability of the EE-form in the intestinal lumen was because this form is hard for pancreatic lipase for digest (Ikeda et al., 1995). There are also opposing opinions that the EE-form and the TG-form of $n$-3 PUFAs exhibited the same effect as increased $n$-3 PUFAs concentrations in human plasma (Krokan et al., 1993). In addition, the effects of the TG-form, EE-form, free acid-form, and PL-form of EPA and DHA on lipid metabolism in animals have been compared and examined (Tang et al., 2012). The effects of different dietary forms of EPA and DHA on bioavailability are still controversial.

The food industry already supplies many kinds of food products fortified with n-3 PUFAs. The EE-form of fish oil is used as supplements in Europe, the United States, and elsewhere, and various degrees of purified $n-3$ PUFAs-EE are on the market. Various public organizations have been recommending an intake of $1 \mathrm{~g} /$ day of long-chain n-3 PUFAs for anti-atherosclerotic and anti-arrhythmic purposes, or 2-4 g/day for a lipid lowering effect (Kris-Ethetron et al., 2003; Kris-Ethetron et al., 2002). Therefore, to intake the desired quantity of long-chain $n$-3 PUFAs using a low concentration $n$-3 PUFA supplement, it is necessary to consume a large quantity of such a supplement. However, there is no definitive information concerning the effects of different degrees of purified DHA-EE on fatty acid metabolism. Therefore, we evaluated the effects of non-purified DHA-EE (L-DHAEE) and highly purified DHA-EE (H-DHAEE) on lipid metabolism and apparent absorption of DHA. In experiment 1, serum and liver lipid contents, fatty acid compositions of liver and brain, excretion of fecal fatty acid-EE, and mRNA expression levels of carboxyl esterase-II (CES-II) in rats fed experiment diets for 4 weeks were analyzed. In experiment 2, serum and liver lipid contents, excretion of fecal fatty acid-EE, fatty acid compositions of liver and white adipose tissue (WAT), and liver fatty acid-metabolizing enzyme activities in rats fed experiment diets for 8 weeks were analyzed.

\section{Method}

\subsection{Materials}

L-DHAEE, H-DHAEE, and soybean oil were supplied as a commercial product from Bizen Chemical Co., Ltd. (Okayama, Japan). EE purified forms of L-DHAEE and H-DHAEE were greater than 99\% pure. Cornstarch, dextrinized cornstarch, cellulose, sucrose, AIN-93 vitamin mixture, AIN-93G mineral mixture, and casein were purchased from Oriental Yeast Co., Ltd. (Tokyo, Japan). Choline bitartrate, L-cystine, acetyl-CoA, palmitoyl-CoA, glucose-6-phosphate, malonyl-CoA, L-carnitine, L-malic acid, flavin adenine dinucleotide, nicotinamide adenine dinucleotide were purchased from Wako Pure Chemical Industries, Ltd. (Osaka, Japan). All other chemicals were obtained from commercial sources and were of reagent grade.

\subsection{Animal Care and Diets}

Male, 5-week-old Wistar rats obtained from Japan SLC, Inc. (Shizuoka, Japan). Rats weighting approximately $130 \mathrm{~g}$ (experiment 1) and $140 \mathrm{~g}$ (experiment 2) were kept in an air-conditioned room (humidity, 60 $\pm 5 \%$; temperature, $22 \pm 1{ }^{\circ} \mathrm{C}$; lights on, 08:00-20:00) with free access to feed and water. The animal experimental protocol was examined and approved by the Animal Ethics Committee of Kansai Medical University and followed the "Guide for the Care and Use of Experimental Animals" issued by the Prime Minister's Office of Japan. Rats were divided to three dietary groups (experiment 1) and four dietary groups (experiment 2) of seven animals per group. Table 1 shows the composition of each experimental diet prepared based on the AIN-93G formulation. Table 2 shows the composition of fatty acids EE in the L-DHAEE- (L-EE) and H-DHAEE (H-EE)-containing diets, as determined by analysis using an Omegawax 250 column (Supelco; Bellefonte, PA, USA) with a gas-liquid chromatography (GLC) system (GC-14B, Shimadzu Co.; Kyoto, Japan). Table 3 shows the composition of fatty acids in the experimental diets, determined by GLC after methylation with sodium methoxide (Prevot \& Mordret, 1976). Injection and detection temperatures were $250{ }^{\circ} \mathrm{C}$ and $260{ }^{\circ} \mathrm{C}$, respectively. The temperature of the column was increased from $120^{\circ} \mathrm{C}$ to $240{ }^{\circ} \mathrm{C}$ at a rate of $2{ }^{\circ} \mathrm{C} / \mathrm{min}$. The amounts of individual fatty acids in the liver and brain (experiment 1) were quantified using C17:0 as an internal standard. 
Fatty acid species were identified by the retention time of standards (Supelco ${ }^{\circledR} 37$ Component FAME Mix, Sigma-Aldrich Japan Co. Ltd.). The DHA-EE contents of the L-EE and H-EE diets (experiment 1) or 2.8\% L-EE (L-EE2.8) and 1.0\% H-EE (H-EE1.0) diets (experiment 2) and the EE contents of 1.0\% L-EE (L-EE1.0) and H-EE1.0 diets (experiment 2) were equal. The differences between the fatty acid compositions of the two experiments were caused by technical dispersion at the time of the preparation of EE.

Table 1. The composition of the experimental diets in experiments 1 and $2(\mathrm{~g} / \mathrm{kg}$ diet $)$

\begin{tabular}{lllllllll}
\hline & \multicolumn{2}{l}{ Experiment 1} & & \multicolumn{2}{l}{ Experiment 2} \\
\cline { 2 - 3 } Components & Control & L-EE & H-EE & & Control & L-EE1.0 & L-EE2.8 & H-EE1.0 \\
\hline Soybean oil & 70.0 & 41.7 & 60.0 & & 70.0 & 60.0 & 42.2 & 60.0 \\
L-DHAEE & - & 28.3 & - & & - & 10.0 & 27.8 & - \\
& - & - & 10.0 & & - & - & - & 10.0 \\
tert-Butylhydroquinone & 0.014 & 0.014 & 0.014 & & 0.014 & 0.014 & 0.014 & 0.014 \\
Choline bitartrate & 2.5 & 2.5 & 2.5 & & 2.5 & 2.5 & 2.5 & 2.5 \\
Dextrinized corn starch & 132 & 132 & 132 & & 132 & 132 & 132 & 132 \\
Corn starch & 397.5 & 397.5 & 397.5 & & 397.5 & 397.5 & 397.5 & 397.5 \\
Sucrose & 100 & 100 & 100 & & 100 & 100 & 100 & 100 \\
Casein & 200 & 200 & 200 & & 200 & 200 & 200 & 200 \\
L-cystine & 3 & 3 & 3 & & 3 & 3 & 3 & 3 \\
Cellulose & 50 & 50 & 50 & & 50 & 50 & 50 & 50 \\
AIN-93G mineral mixture & 35 & 35 & 35 & & 35 & 35 & 35 & 35 \\
AIN-93 vitamin mixture & 10 & 10 & 10 & & 10 & 10 & 10 & 10 \\
\hline
\end{tabular}

Diets were prepared based on the AIN-93G formulation.

Table 2. Fatty acid ethyl ester composition of L-DHAEE and H-DHAEE in experiments 1 and 2 (wt $\%$ )

\begin{tabular}{|c|c|c|c|c|c|}
\hline \multirow{2}{*}{\multicolumn{2}{|c|}{ Fatty acid }} & \multicolumn{2}{|c|}{ Experiment 1} & \multicolumn{2}{|c|}{ Experiment 2} \\
\hline & & \multirow{2}{*}{$\begin{array}{l}\text { L-DHAE } \\
\text { F } \\
26.3\end{array}$} & \multirow[t]{2}{*}{ H-DHAEE } & \multirow{2}{*}{$\begin{array}{l}\text { L-DHAEE } \\
24.6\end{array}$} & \multirow[t]{2}{*}{ H-DHAEE } \\
\hline $\mathrm{C} 16$ & 0 & & & & \\
\hline $\mathrm{C} 16$ & $1 n-7$ & 5.1 & & 5.2 & \\
\hline $\mathrm{C} 18$ & 0 & 7.1 & 1.3 & 6.6 & \\
\hline $\mathrm{C} 18$ & $1 n-9$ & 13.9 & 2.1 & 12.8 & \\
\hline $\mathrm{C} 18$ & $1 n-7$ & 3.3 & & 2.6 & \\
\hline $\mathrm{C} 18$ & $2 n-6$ & 2.2 & & 1.8 & \\
\hline $\mathrm{C} 18$ & $3 n-3$ & 0.6 & & 1.1 & \\
\hline $\mathrm{C} 20$ & $4 n-6$ & 1.0 & 2.0 & 2.3 & 2.3 \\
\hline $\mathrm{C} 20$ & $5 n-3$ & 3.0 & 3.0 & 6.8 & 7.2 \\
\hline $\mathrm{C} 22$ & $5 n-3$ & 2.2 & 5.8 & 2.0 & 6.0 \\
\hline $\mathrm{C} 22$ & $5 n-6$ & 1.2 & 3.0 & 2.1 & 3.6 \\
\hline $\mathrm{C} 22$ & $6 n-3$ & 26.7 & 75.4 & 27.6 & 76.9 \\
\hline \multicolumn{2}{|c|}{ Others } & 7.4 & 7.4 & 4.5 & 4.0 \\
\hline
\end{tabular}

C16:0, palmitic acid; C16:1n-7, palmitoleic acid; C18:0, stearic acid; C18:1n-9, oleic acid; C18:1 $n-7$, vaccenic acid; C18:2n-6, linoleic acid; C18:3n-3, $\alpha$-linolenic acid; C20:4n-6, arachidonic acid; C20:5n-3, eicosapentaenoic acid; $\mathrm{C} 22: 5 n-3$, docosapentaenoic acid; C22:5n-6, osbond acid; C22:6n-3, docosahexaenoic acid. 
Table 3. Fatty acid composition of experimental diets in experiments 1 and $2(\mathrm{wt} \%)$

\begin{tabular}{|c|c|c|c|c|c|c|c|c|}
\hline \multirow{2}{*}{\multicolumn{2}{|c|}{ Fatty acid }} & \multicolumn{3}{|c|}{ Experiment 1} & \multicolumn{4}{|c|}{ Experiment 2} \\
\hline & & \multirow{2}{*}{$\begin{array}{l}\text { Control } \\
10.6\end{array}$} & \multirow{2}{*}{$\frac{\text { L-EE }}{16.9}$} & \multirow{2}{*}{$\begin{array}{l}\text { H-EE } \\
9.1\end{array}$} & \multirow{2}{*}{$\begin{array}{l}\text { Control } \\
10.6\end{array}$} & \multirow{2}{*}{$\frac{\text { L-EE1.0 }}{12.6}$} & \multirow{2}{*}{$\frac{\text { L-EE2.8 }}{16.1}$} & \multirow{2}{*}{$\begin{array}{l}\text { H-EE1.C } \\
9.1\end{array}$} \\
\hline $\mathrm{C} 16$ & 0 & & & & & & & \\
\hline $\mathrm{C} 16$ & $: 1 n-7$ & N.D. & 2.1 & N.D. & N.D. & 0.7 & 2.1 & N.D. \\
\hline $\mathrm{C} 18$ & $: 0$ & 3.8 & 5.1 & 3.4 & 3.9 & 4.2 & 4.9 & 3.3 \\
\hline $\mathrm{C} 18$ & $: 1 n-9$ & 21.8 & 18.6 & 19.0 & 22.1 & 20.5 & 18.2 & 18.7 \\
\hline $\mathrm{C} 18$ & $: 1 n-7$ & 1.4 & 2.1 & 1.2 & 1.4 & 1.6 & 1.9 & 1.2 \\
\hline $\mathrm{C} 18$ & $: 2 n-6$ & 53.7 & 32.9 & 46.0 & 54.2 & 46.3 & 33.1 & 46.0 \\
\hline $\mathrm{C} 18$ & $: 3 n-3$ & 5.8 & 3.7 & 5.0 & 5.8 & 5.1 & 3.9 & 5.0 \\
\hline $\mathrm{C} 20$ & $: 4 n-6$ & N.D. & 0.4 & 0.3 & N.D. & 0.3 & 0.9 & 0.3 \\
\hline $\mathrm{C} 20$ & $: 5 n-3$ & N.D. & 1.2 & 0.4 & N.D. & 1.0 & 2.7 & 1.0 \\
\hline $\mathrm{C} 22$ & $: 5 n-3$ & N.D. & 0.9 & 0.8 & N.D. & 0.3 & 0.8 & 0.9 \\
\hline $\mathrm{C} 22$ & $: 5 n-6$ & N.D. & 0.5 & 0.4 & N.D. & 0.3 & 0.8 & 0.5 \\
\hline $\mathrm{C} 22$ & $: 6 n-3$ & N.D. & 10.8 & 10.8 & N.D. & 3.9 & 11.0 & 11.0 \\
\hline Other & & 2.9 & 4.7 & 3.6 & 2.0 & 3.2 & 3.6 & 3.1 \\
\hline
\end{tabular}

N.D., not detected.

Rats were fed on the experimental diets for 28 days (experiment 1) or 56 days (experiment 2). Body weight (BW) and food consumption were recorded daily. Feces were collected daily from each group at 9:00 am for 7 days before sacrifice. At the end of the experimental period, rats were weighed and then sacrificed under pentobarbital anesthesia between 08:00 and 10:00. Rats were not deprived of food prior to being sacrificed, because food deprivation causes to a significant decrease in the expression of genes involved in lipid metabolism (Horton et al., 1998). Blood was collected without the use of anti-coagulant, and serum was separated by centrifugation at 2,000 $\mathrm{g}$ for $15 \mathrm{~min}$, and stored at $-70^{\circ} \mathrm{C}$ until analysis. Brain, liver, and abdominal WAT (epididymal, mesenteric, and perirenal) were removed rapidly, then weighed, rinsed with ice-cold saline, and frozen in liquid nitrogen, followed by storage at $-70{ }^{\circ} \mathrm{C}$ until analysis. In experiment 1 , the small intestine was excised, rinsed with $0.9 \%$ $\mathrm{NaCl}$, and divided into two equal lengths (jejunum and ileum regions). The jejunum section mucosa was scraped off, and was stored in RNA-Later Storage Solution (Sigma-Aldrich Japan Co. Ltd.) for mRNA expression analysis.

\subsection{Analysis of Biochemical Tests and Lipids}

Serum biochemical tests were conducted by using an Olympus AU5431 automatic analyzer (Olympus Co., Tokyo, Japan). Liver total lipids were extracted by the method of Bligh and Dyer (1959), and the solvent evaporated and total lipids weighted. Each total lipid sample was resolved in an equal volume of dimethylsulfoxide, and the TG content was quantified using an enzymatic assay kit (Triglyceride E-Test Wako, Wako Pure Chemical Industries Ltd.). Liver cholesterol content was determined using a GLC system (GC-14B, Shimadzu Co.) with an SE-30 column (Shinwa Chemical Industries; Kyoto, Japan), in which internal standard ( $5 \alpha$-cholestane) was used. Liver PL content was quantified by phosphorus analysis (Rouser et al., 1970).

\subsection{Analysis of mRNA Expression (Experiment 1)}

Mucosal total RNA from the jejunum was extracted using TRIzol reagent (Invitrogen; Carlsbad, CA, USA). Total RNA was reverse transcribed using a RevaTraAce ${ }^{\circledR}$ qPCR RT kit (Toyobo Co. Ltd.; Osaka, Japan) for cDNA synthesis. Real-time quantitative PCR was carried out with a DNA Engine Opticon 2 (Bio-Rad Laboratories; Hercules, CA, USA) using GoTaq ${ }^{\circledR}$ qPCR Master Mix (Promega; Madison, WI, USA). Primer sequences used for the detection of glyceraldehyde 3-phosphate dehydrogenase (GAPDH) and CES-II were as follows: forward: 5'-ATGACTCTACCCACGGCAAG-3' and reverse: 5'-TACTCAGCACCAGCATCACC-3'; for GAPDH, forward: 5'-GGTGGTTGTCGCTATCCAGT-3' and reverse: 5'-CAGCCACTTGGTCCAGGTAT-3'; for CES-II, which were designed using GENETYX-MAC (version 13.0.4, Genetyx; Tokyo, Japan). CES-II level was expressed after normalization to the GAPDH expression level.

\subsection{Analysis of Hepatic Enzyme Activity (Experiment 2)}

Hepatic crude, mitochondria, and cytosol fractions were prepared using an ultracentrifuge. Acyl-CoA oxidase 
(ACOX) activity in the crude fraction was measured by the method of Ide et al. (1987). Carnitine palmitoyl transferase-II (CPT-II) activity in mitochondria fraction was measured by the method of Markwell et al. (1973). Cytosol acetyl-CoA carboxylase (Tanabe et al., 1981), Fatty acid synthase (Kelley et al., 1986), and glucose-6-phosphate dehydrogenase (G6PDH) (Kelley et al., 1984) activities were measured spectrophotometrically. The protein content of each hepatic fraction was measured by the method of Lowry et al. (1951) using bovine serum albumin as a standard.

\subsection{Analysis of Fatty Acid Composition in Organs}

Liver, brain (experiment 1), and WAT (experiment 2) total lipids were extracted by the method of Bligh and Dyer (1959). Total lipid fatty acid composition was measured using an Omegawax $250^{\circledR}$ column (Supelco Co., Ltd.) with a GLC system (GC-14B, Shimadzu Co.; Kyoto, Japan). Fatty acids were methylated using sodium methoxide. Injection and detection temperatures were $250{ }^{\circ} \mathrm{C}$ and $260{ }^{\circ} \mathrm{C}$, respectively. The temperature of the column was increased from $120^{\circ} \mathrm{C}$ to $240^{\circ} \mathrm{C}$ at a rate of $2{ }^{\circ} \mathrm{C} / \mathrm{min}$. The amounts of individual fatty acids in the liver and brain (experiment 1) were quantified using $\mathrm{C} 17: 0$ as an internal standard. Fatty acid species were identified by the retention time of standards (Supelco ${ }^{\circledR} 37$ Component FAME Mix, Sigma-Aldrich Japan Co. Ltd.).

Fecal lipids (experiments 1 and 2) were extracted by the method of Bligh and Dyer (1959), and then the EE fraction was separated using a silica gel Sep-Pak ${ }^{\circledR}$ cartridge column (Nihon Waters K.K. Tokyo, Japan) with chloroform and methanol as elution solvents. The individual fatty acid EEs of fecal lipids was measured using an Omegawax $250^{\circledR}$ column (Supelco Co., Ltd.) with a GLC mass spectrometer (GC-MS QP2010, Shimadzu Co.). The amount of individual fatty acid EEs was quantified using C17:0-EE as an internal standard, and the fatty acid EE species were identified using standard components and mass spectra.

\subsection{Statistical Analysis}

Data are presented as means and standard error of the mean of seven rats. Statistical differences between multiple groups were determined using analysis of variance. Statistical comparisons were conducted by applying the Tukey-Kramer test, and significant differences were decided at $p<0.05$. Analyses were conducted using StatView-J software (version 5.0, Abacus Concepts; CA, USA).

\section{Results and Discussion}

\subsection{Growth Parameter and Organ Weight}

Tables 4 and 5 show the growth parameters and results of serum biochemical tests, liver lipid contents, and fecal EE excretion contents in experiments 1 and 2, respectively. In experiment 1, there were no significant differences in BW gain or food intake among the groups. In experiment 2, the food intake of the L-EE2.8 group was significantly higher than the control group, and the BW gain of the L-EE2.8 group tended to be higher compared with the other groups. The food efficiency was not different among the groups, thus the tendency for the increase in the BW gain was caused by the increase in food intake. In experiments 1 and 2, the relative liver weight and each WAT weight were not changed among the groups. 
Table 4. Effect of experimental diets on growth parameters and results of serum biochemical tests, liver lipid contents, and fecal EE contents in experiment 1

\begin{tabular}{lllllllll}
\hline & Control & & L-EE & & H-EE & \\
& & & & & & & \\
\hline Growth parameter & & & & & & & \\
$\quad$ BW gain (g/day) & 6.2 & \pm 0.1 & 6.2 & \pm 0.1 & 6.1 & \pm & 0.2 \\
$\quad$ Food intake (g/day) & 17.3 & \pm 0.8 & 17.8 & \pm 0.8 & 17.9 & \pm & 0.8 \\
Organ weight (g/100 g BW) & & & & & & & \\
$\quad$ Liver weight & 3.63 & \pm 0.06 & 3.77 & \pm 0.04 & 3.77 & \pm & 0.06 \\
Total WAT weight ${ }^{\dagger}$ & 3.66 & \pm 0.18 & 3.57 & \pm 0.09 & 3.67 & \pm & 0.18 \\
Serum & & & & & & & \\
Total protein (mg/dL) & 5.44 & \pm 0.06 & 5.30 & \pm 0.09 & 5.37 & \pm & 0.10 \\
A/G & 2.13 & \pm 0.06 & 2.28 & \pm 0.04 & 2.37 & \pm & 0.09 \\
AST (IU/L) & 77.9 & $\pm 2.5^{\mathrm{a}}$ & 80.4 & $\pm 3.1^{\mathrm{ab}}$ & 87.9 & \pm & $1.7^{\mathrm{b}}$ \\
ALT (IU/L) & 46.4 & \pm 2.1 & 47.0 & \pm 1.3 & 51.0 & \pm & 2.7 \\
SUN (mg/dL) & 16.7 & \pm 0.8 & 16.9 & \pm 1.1 & 17.4 & \pm & 0.9 \\
Total lipid (mg/dL) & 175.6 & \pm 7.3 & 155.3 & \pm 4.6 & 156.0 & \pm & 6.6 \\
PL (mg/dL) & 101.7 & \pm 6.1 & 94.6 & \pm 3.0 & 95.4 & \pm & 4.2 \\
Cholesterol (mg/dL) & 61.4 & $\pm 1.9^{\mathrm{b}}$ & 50.9 & $\pm 2.8^{\mathrm{a}}$ & 51.1 & \pm & $2.8^{\mathrm{a}}$ \\
TG (mg/dL) & 26.8 & \pm 1.2 & 23.6 & \pm 1.3 & 23.7 & \pm & 0.9 \\
Liver (mg/g) & & & & & & & \\
Total lipid & 60.2 & \pm 4.4 & 48.0 & \pm 1.6 & 57.1 & \pm & 2.5 \\
PL & 28.9 & \pm 0.6 & 28.0 & \pm 0.8 & 28.5 & \pm & 0.9 \\
Cholesterol & 3.61 & \pm 0.21 & 3.47 & \pm 0.15 & 3.35 & \pm & 0.36 \\
TG & 29.8 & $\pm 3.4^{\mathrm{b}}$ & 16.6 & $\pm 1.5^{\mathrm{a}}$ & 25.2 & \pm & $2.3^{\mathrm{ab}}$ \\
Faces (mg/day) & & & & & & & \\
DHA-EE & N.D. & & & 0.62 & $\pm 0.05^{\mathrm{b}}$ & 0.43 & \pm & $0.01^{\mathrm{a}}$ \\
Total EE & N.D. & & 4.08 & $\pm 0.37^{\mathrm{b}}$ & 3.19 & \pm & $0.21^{\mathrm{a}}$ \\
\hline
\end{tabular}

Data represent means \pm SEM $(\mathrm{n}=7)$. Values in the same row not sharing a common superscript are significantly different at $p<0.05$ using the Tukey-Kramer test.

${ }^{\dagger}$ Total WAT represents the sum of the epididymal, mesenteric, perirenal, and retroperitoneal WAT weights.

A/G, albumin/globulin; ALT, alanine aminotransferase; AST, aspartate aminotransferase; SUN, serum urea nitrogen. 
Table 5. Effect of experimental diets on growth parameters and the results of serum biochemical tests, liver lipid contents, and fecal EE contents in experiment 2

\begin{tabular}{|c|c|c|c|c|c|c|c|c|c|c|c|c|}
\hline & \multicolumn{3}{|c|}{ Control } & \multicolumn{3}{|c|}{ L-EE1.0 } & \multicolumn{3}{|c|}{ L-EE2.8 } & \multicolumn{3}{|c|}{ H-EE1.0 } \\
\hline \multicolumn{13}{|l|}{ Growth parameter } \\
\hline BW gain (g/day) & 4.99 & \pm & 0.23 & 4.99 & \pm & 0.27 & 5.32 & \pm & 0.10 & 4.92 & \pm & 0.12 \\
\hline Food intake (g/day) & 20.2 & \pm & $0.87^{\mathrm{a}}$ & 20.9 & \pm & $0.96^{\mathrm{ab}}$ & 22.0 & \pm & $0.83^{b}$ & 20.4 & \pm & $0.86^{\mathrm{at}}$ \\
\hline Food efficiency $(\mathrm{g} / \mathrm{kcal})^{\dagger}$ & 0.062 & \pm & 0.003 & 0.060 & \pm & 0.003 & 0.062 & \pm & 0.002 & 0.061 & \pm & 0.002 \\
\hline \multicolumn{13}{|l|}{ Organ weight ( $\mathrm{g} / 100 \mathrm{~g} \mathrm{BW})$} \\
\hline Liver weight & 3.23 & \pm & 0.08 & 3.10 & \pm & 0.08 & 3.21 & \pm & 0.07 & 3.16 & \pm & 0.05 \\
\hline Epididymal WAT weight & 1.43 & \pm & 0.08 & 1.40 & \pm & 0.08 & 1.50 & \pm & 0.06 & 1.30 & \pm & 0.05 \\
\hline Mesentery WAT weight & 1.14 & \pm & 0.08 & 1.19 & \pm & 0.07 & 1.38 & \pm & 0.13 & 1.15 & \pm & 0.10 \\
\hline $\begin{array}{lr}\text { Perirenal } & \text { and } \\
\text { retroperitoneal } & \text { WAT } \\
\text { weight } & \end{array}$ & 2.02 & \pm & 0.21 & 1.75 & \pm & 0.16 & 2.08 & \pm & 0.23 & 1.71 & \pm & 0.17 \\
\hline \multicolumn{13}{|l|}{ Serum } \\
\hline AST (IU/L) & 97.6 & \pm & 4.0 & 94.9 & \pm & 3.0 & 93.9 & \pm & 3.0 & 99.6 & \pm & 3.6 \\
\hline ALT (IU/L) & 54.4 & \pm & 3.4 & 49.1 & \pm & 2.9 & 51.9 & \pm & 1.9 & 55.9 & \pm & 2.8 \\
\hline SUN (mg/dL) & 17.1 & \pm & 0.7 & 18.3 & \pm & 1.0 & 16.1 & \pm & 0.5 & 16.6 & \pm & 0.6 \\
\hline Total lipid (mg/dL) & 272.0 & \pm & $7.9^{\mathrm{a}}$ & 236.6 & \pm & $7.0^{\mathrm{b}}$ & 226.1 & \pm & $4.2^{\mathrm{b}}$ & 213.3 & \pm & $9.0^{\mathrm{b}}$ \\
\hline PL (mg/dL) & 141.9 & \pm & $3.8^{\mathrm{b}}$ & 126.9 & \pm & $3.9^{\mathrm{a}}$ & 124.0 & \pm & $2.2^{\mathrm{a}}$ & 114.7 & \pm & $4.1^{\mathrm{a}}$ \\
\hline Cholesterol (mg/dL) & 83.3 & \pm & $3.3^{\mathrm{b}}$ & 71.6 & \pm & $2.2^{\mathrm{a}}$ & 73.6 & \pm & $2.1^{a b}$ & 70.9 & \pm & $3.8^{\mathrm{a}}$ \\
\hline $\mathrm{TG}(\mathrm{mg} / \mathrm{dL})$ & 42.3 & \pm & $2.1^{\mathrm{b}}$ & 35.9 & \pm & $2.2^{b}$ & 26.0 & \pm & $1.4^{\mathrm{a}}$ & 26.7 & \pm & $1.3^{\mathrm{a}}$ \\
\hline \multicolumn{13}{|l|}{ Liver $(\mathrm{mg} / \mathrm{g})$} \\
\hline Total lipid & 53.7 & \pm & 7.2 & 52.6 & \pm & 6.1 & 41.1 & \pm & 3.3 & 41.4 & \pm & 3.1 \\
\hline PL & 23.4 & \pm & 1.1 & 24.3 & \pm & 1.1 & 24.9 & \pm & 1.1 & 24.1 & \pm & 0.8 \\
\hline Cholesterol & 2.36 & \pm & 0.15 & 2.33 & \pm & 0.11 & 2.11 & \pm & 0.09 & 2.45 & \pm & 0.20 \\
\hline TG & 20.9 & \pm & 1.4 & 21.2 & \pm & 3.3 & 18.2 & \pm & 1.2 & 18.0 & \pm & 2.8 \\
\hline \multicolumn{13}{|l|}{ Faces (mg/day) } \\
\hline DHA-EE & N.D. & & & 0.43 & \pm & $0.03^{\mathrm{a}}$ & 2.38 & \pm & $0.08^{b}$ & 0.59 & \pm & $0.03^{\mathrm{a}}$ \\
\hline Total EE & N.D. & & & 2.55 & \pm & $0.34^{\mathrm{a}}$ & 4.06 & \pm & $0.33^{\mathrm{b}}$ & 2.92 & \pm & $0.20^{\mathrm{a}}$ \\
\hline
\end{tabular}

Data represent means \pm SEM $(\mathrm{n}=7)$. Values in the same row not sharing a common superscript are significantly different at $p<0.05$ using the Tukey-Kramer test.

${ }^{\dagger} \mathrm{BW}$ gain (g/day) / energy intake (kcal/day)

\subsection{Biochemical Tests}

It has been suggested that fatty acid EEs are ingested as fatty acids and ethanol, thus the intake of a large amount fatty acid EEs might lead to ethanol toxicity (Tang et al., 2012). Ethanol is mostly metabolized in the liver, and ethanol over-consumption causes liver diseases such as fatty liver, hepatitis, and cirrhosis. The L-EE (experiment 1) and L-EE2.8 (experiment 2) groups, which have the highest abundance of fatty acid EEs, did not have different serum aspartate aminotransferase (AST) and alanine aminotransferase (ALT) levels, which acts as an index for liver damage, compared with the control group (Table 4 and 5). In addition, the L-EE and L-EE2.8 diets did not increase the liver total lipid content compared with other diets in experiments 1 and 2. Therefore, there was no influence of ethanol toxicity at $27.8 \mathrm{~g} / \mathrm{kg}$ fatty acid EE content using L-DHAEE in the present study. In experiment 1 , the H-EE group had significantly higher serum AST and a tendency for higher serum ALT. The reason for the increasing serum AST and ALT levels in response to dietary H-EE diets was not clear. Serum total protein, albumin/globulin, PL, and serum urea nitrogen levels were not affected by DHA-EE administration in experiments 1 and 2 .

After 8 weeks feeding of L-DHAEE and H-DHAEE, serum total lipid and TG contents in rats fed the L-EE2.8 
and H-EE1.0 diets were significantly decreased compared with rats fed the control diets (Table 5). Whereas after 4 weeks feeding of DHA-EE, serum TG contents in the L-EE and H-EE groups tended to be lower than the control group, but not to a significant degree (Table 4). One of the health-promoting effects of DHA is a decrease in serum TG content (Berge et al., 1999). The present results suggested that DHA-EE consumption was efficacious in decreasing serum TG contents; however, serum TG contents were not different between rats fed L-DHAEE and those fed H-DHAEE.

In experiments 1 and 2, serum cholesterol contents in rats fed DHA-EE-containing diets were significantly decreased compared with the control groups. Whereas, the consumption of DHA-EE did not affect the liver cholesterol content in experiments 1 and 2. Frøyland et al. also showed that intake of fish oil containing DHA inhibits hepatic 3-hydroxy-3-methylglutaryl-CoA reductase, a rate-limiting enzyme in cholesterol synthesis, activity, (Frøyland et al., 1996).

\subsection{Apparent Absorption of DHA-EE}

The absorption of DHA in the intestine was lower as an EE form than as the free acid form of TG (Ikeda et al., 1993). To compare bioavailability between L-DHAEE and H-DHAEE, we then examined DHA-EE and total EE excretion in feces as the apparent rate of DHA-EE absorption. In experiments 1 and 2, groups receiving L-DHAEE in the diets at a level of about 2.8\% (L-EE and L-EE2.8) had significantly higher fecal DHA-EE and total EE excretion contents compared with the groups receiving L-DHAEE or H-DHAEE at a level of about $1.0 \%$ (H-EE, L-EE1.0, and H-EE1.0 groups). Between the L-EE and H-EE or L-EE2.8 and H-EE1.0 groups with an equal DHA-EE content in the diets, H-DHAEE at a level of 1.0\% (H-EE and H-EE1.0 groups) had significantly higher apparent DHA-EE absorption than the diets with L-DHAEE at a level of about 2.8\% (L-EE and L-EE2.8 groups). These results showed that reduced apparent EE absorption occurred with the increase in the ratio of EEs in the diet. Therefore, dietary H-DHAEE was more efficacious in making DHA available in the body than dietary L-DHAEE. It is known that the bioavailability of fatty acid EEs changed in low- or high-fat diet conditions (Lawson \& Hughes, 1988). Because we used low-fat diet conditions in the present study, comparison of low and high concentrations of DHA-EE in high-fat diet conditions will also be required.

\subsection{CES-II Expression Level of in the Jejunum (Experiment 1)}

Fatty acid EEs are difficult to hydrolyze in the intestinal lumen because they are poor substrates for pancreatic lipase, thus they are hydrolyzed in intestinal epithelial cells (Reicks et al., 1990; Nelson \& Ackman, 1988). Therefore, this study evaluated the mRNA expression levels of CES-II in the jejunum using quantitative PCR in experiment 1 . The CES-II mRNA expression levels were unchanged among the groups (data not shown). This result implies that the reduction in apparent DHA absorption was not related to hydrolyzation in intestinal epithelial cells.

\subsection{Fatty acid-Metabolizing Enzyme Activities in the Liver (Experiment 2)}

Berge et al. showed that dietary DHA can alter the activities of enzymes related to endogenous fatty acid metabolism (Berge et al., 1999). To investigate the regulation of fatty acid metabolism, we analyzed the effect of dietary L-DHAEE and H-DHAEE on the activities of hepatic enzymes related to fatty acid synthesis and oxidation in experiment 2 . Table 6 shows the activities of liver fatty acid metabolism-related enzymes. ACOX, a key enzyme of fatty acid peroxisomal $\beta$-oxidation, activity was markedly increased in rats fed the H-EE1.0 diet compared with rats fed the control, L-EE1.0, or L-EE2.8 diets, and it tended to be higher in rats fed L-DHAEE-containing diets than in rats fed the control diet (Table 6). CPT-II, a key enzyme of fatty acid mitochondrial $\beta$-oxidation, activity was not changed among the groups. The reason for the different ACOX activities between the L-EE2.8 and H-EE groups might be caused by the apparent level of DHA absorption. A previous study suggested that EPA stimulates $\beta$-oxidation in mitochondria, whereas DHA is more efficacious for $\beta$-oxidation in peroxisomes. In the present study, EPA contents in the DHA-EE-containing diet were low (about $0.8 \%$ of total fat), thus, CPT-II activity was unchanged. On the other hand, fatty acid synthase, a key enzyme in the regulation of de novo fatty acid synthesis, and malic enzyme, a key enzyme of the production of NADPH in cell, activities were not significantly change among the groups. G6PDH, a key enzyme of the production of NADPH in cell, and of the regulating fatty acids and cholesterol biosynthesis, activity tended to be lower in the L-EE2.8 and H-EE1.0 groups compared with the control and L-EE1.0 groups. The decreased serum TG content in the DHA-EE-containing diets may be in part caused by the inhibition of G6PDH and the increase of fatty acid oxidation in peroxisome. 
Table 6. Activities of liver fatty acid metabolism-related enzymes in experiment 2 (nmol/min/mg protein)

\begin{tabular}{lllllllllll}
\hline & \multirow{2}{*}{ Control } & \multicolumn{3}{c}{ L-EE1.0 } & \multicolumn{3}{c}{ L-EE2.8 } & \multicolumn{3}{c}{ H-EE1.0 } \\
\hline FAS & 1.80 & \pm & 0.17 & 1.99 & \pm 0.14 & 2.22 & \pm 0.17 & 2.06 & \pm 0.23 \\
ACC & 242.4 & \pm & 14.5 & 269.9 & \pm 11.6 & 267.6 & \pm & 14.8 & 224.4 & \pm 10.9 \\
G6PDH & 130.1 & \pm & 11.1 & 134.6 & \pm 12.5 & 80.3 & \pm & 11.4 & 105.9 & \pm 10.7 \\
ME & 8.69 & \pm & 0.98 & 9.34 & \pm 0.88 & 9.04 & \pm & 1.40 & 12.28 & \pm 0.76 \\
CPT-II & 4.88 & \pm & 0.52 & 5.47 & \pm 0.28 & 4.86 & \pm 0.27 & 5.40 & \pm 0.23 \\
ACOX & 3.68 & \pm & 0.20 & 4.31 & $\pm 0.40^{\text {a }}$ & 4.53 & $\pm 0.36^{\text {a }}$ & 6.60 & $\pm 0.29^{\mathrm{b}}$ \\
\hline
\end{tabular}

Data represent means \pm SEM $(\mathrm{n}=7)$. Values in the same row not sharing a common superscript are significantly different at $p<0.05$ using the Tukey-Kramer test.

ACC, acetyl-CoA carboxylase; FAS, fatty acid synthase; ME, malic enzyme.

\subsection{Fatty acid Composition in Organs}

Figure 1 (experiment 1 ) and Figure 2 (experiment 2 ) show the main fatty acids present in the liver. In experiment 1, the contents of C16:0, C16:1n-9, C18:0, C18:1n-9, and C18:2n-6 in the liver were decreased in the L-EE group compared with the control and H-EE groups, and the decreased fatty acid contents seen with dietary L-DHAEE might be associated with decreased liver TG contents. Liver DHA content of the H-EE group, compared with L-EE group was increasing in experiment 1; however, this was not different between the L-EE2.8 and H-EE1.0 groups in experiment 2. When DHA-EE was fed to rats for 4 weeks, H-DHAEE was more efficacious than L-DHAEE at increasing liver DHA contents owing to the enhancement of apparent DHA absorption. Brain fatty acid contents of rats fed DHA-EE-contained diets were found to have no significant change after 4 weeks (data not shown).

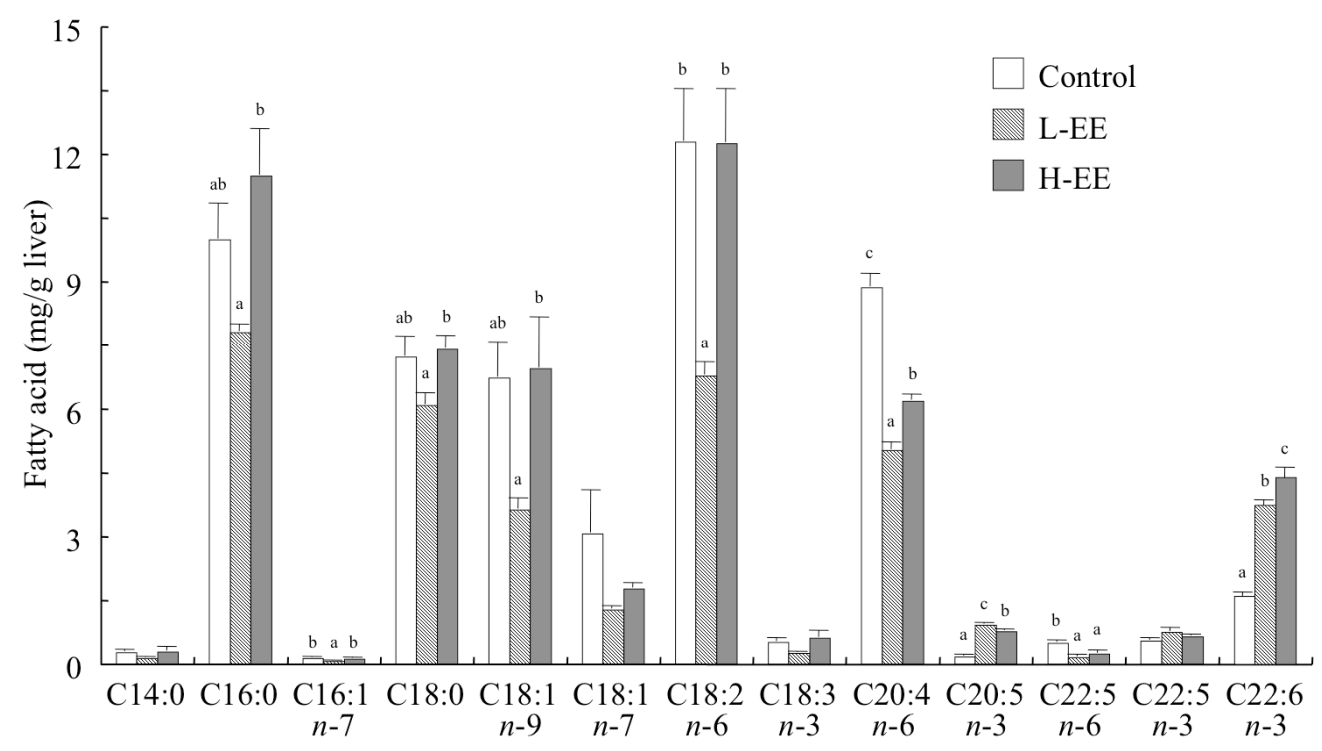

Figure 1. Amounts of main fatty acids in the liver in experiment 1

Data represent means \pm SEM $(\mathrm{n}=7)$. Values in the same row not sharing a common superscript are significantly different at $p<0.05$ using the Tukey-Kramer test.

C14:0, myristic acid; C16:0, palmitic acid; C16:1n-9, palmitoleic acid; C18:0, stearic acid; C18:1n-9, oleic acid; C18:1n-7, vaccenic acid; C18:2n-6, linoleic acid; C18:3 n-3, $\alpha$-linolenic acid; C20:4n-6, arachidonic acid; $\mathrm{C} 20: 5 n-3$, eicosapentaenoic acid; $\mathrm{C} 22: 5 n-6$, osbond acid; $\mathrm{C} 22: 5 n-3$, docosapentaenoic acid; $\mathrm{C} 22: 6 n-3$, docosahexaenoic acid. 


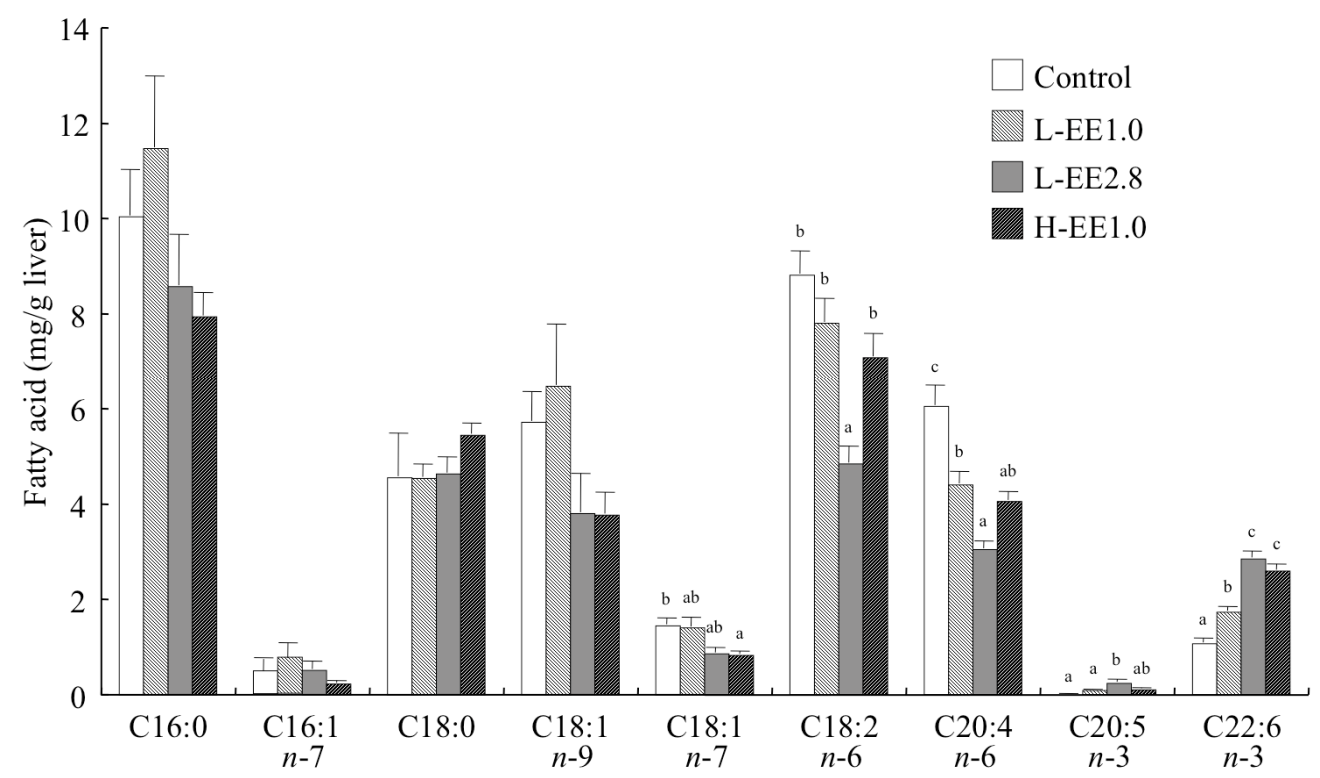

Figure 2. Amounts of main fatty acids in the liver in experiment 2

Data represent means \pm SEM $(n=7)$. Values in the same row not sharing a common superscript are significantly different at $p<0.05$ using a Tukey-Kramer test.

C16:0, palmitic acid; C16:1n-9, palmitoleic acid; C18:0, stearic acid; C18:1n-9, oleic acid; C18:1n-7, vaccenic acid; C18:2n-6, linoleic acid; C20:4n-6, arachidonic acid; C20:5n-3, eicosapentaenoic acid; C22:6n-3, docosahexaenoic acid.

WAT not only has energy-storage functions but also plays an important part in regulating lipid and glucose homeostasis by secreting adipocytokines, including leptin, adiponectin, and tumor necrosis factor- $\alpha$ (Matsuzawa, 2006; Maeda et al., 1997). Dietary DHA regulates adipocytokine gene expression and secretion simultaneously with accumulation of DHA in WAT in animal experiments (Moreno-Aliaga et al., 2010). In addition, dietary DHA induces diverse responses in the relative concentrations of DHA and adipocytokine secretion among the different WATs (Lefils et al., 2010). To clarify the effect of dietary L-DHAEE and H-DHAEE on WAT fatty acid composition, the fatty acid composition of WAT, including epididymal, mesenteric, and perirenal WAT were analyzed. Figure 3 shows the percentages of the main fatty acids in epididymal (A), mesenteric (B), and perirenal (C) WAT total lipids. The relative DHA composition in epididymal, mesenteric, and perirenal WAT between the L-EE2.8 and H-EE1.0 groups did not differ significantly. Epididymal WAT showed the highest relative DHA composition. In addition, the saturated fatty acid composition, including C14:0, C16:0, and C18:0, in the L-EE2.8 group was higher than the other groups. Palmitic acid (C16:0) has a robust effect on the inflammatory capacity of WAT (Kien et al., 2005), induces endoplasmic reticulum stress, (Guo et al., 2007), and enhances the secretion of tumor necrosis factor- $\alpha$ and interleukin-10 in WAT (Bradley et al., 2008). 

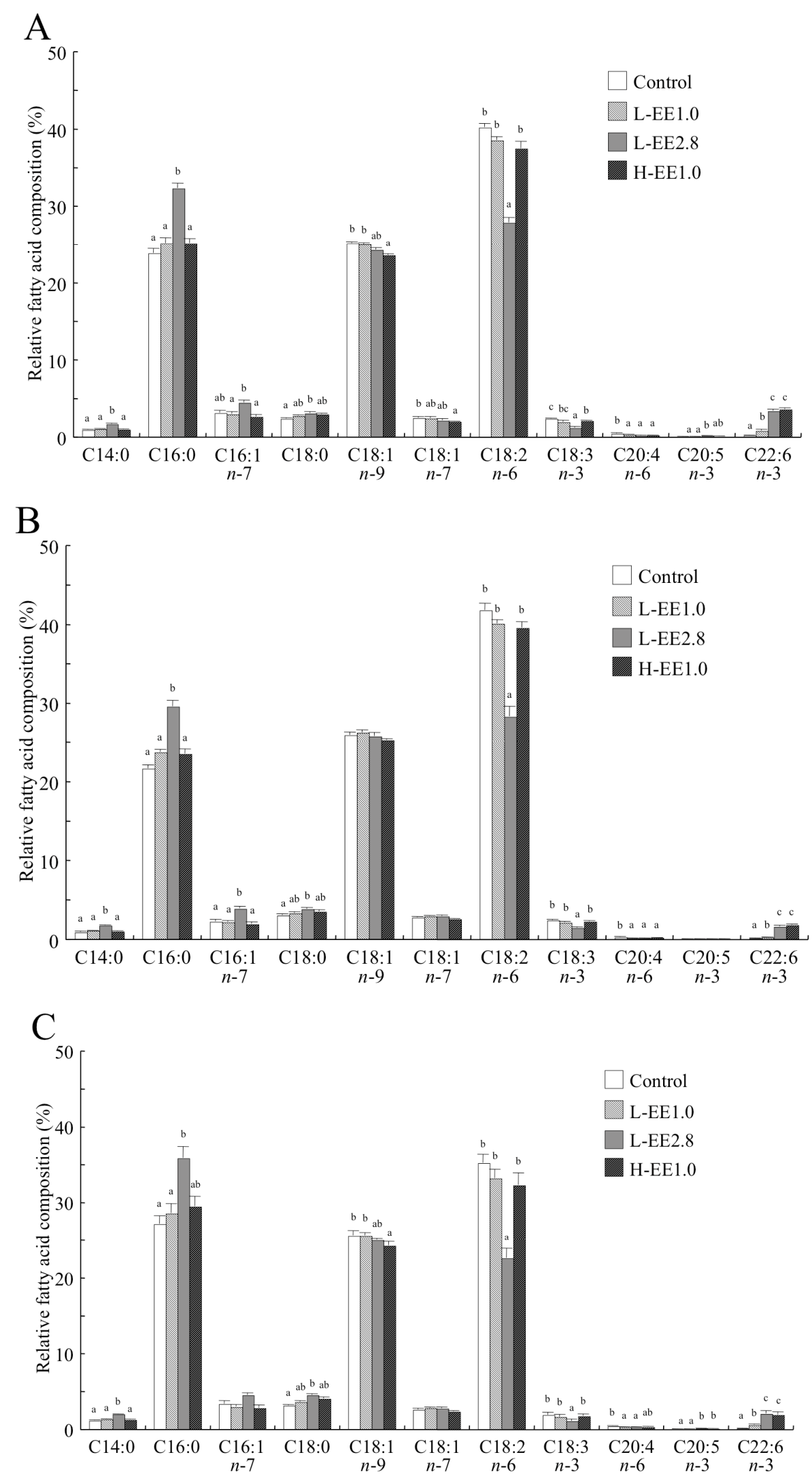

Figure 3. Relative main fatty acid compositions of epididymal (A), mesenteric (B), and perirenal (C) WAT in experiment 2

Data represent means $\pm \operatorname{SEM}(\mathrm{n}=7)$. Values in the same row not sharing a common superscript are significantly different at $p<0.05$ using a Tukey-Kramer test. 
C14:0, myristic acid; C16:0, palmitic acid; C16:1n-9, palmitoleic acid; C18:0, stearic acid; C18:1n-9, oleic acid; C18:1n-7, vaccenic acid; C18:2n-6, linoleic acid; C18:3n-3, $\alpha$-linolenic acid; C20:4n-6, arachidonic acid; $\mathrm{C} 20: 5 n-3$, eicosapentaenoic acid; $\mathrm{C} 22: 6 n-3$, docosahexaenoic acid.

\section{Conclusions}

This study showed that compared with L-DHAEE, H-DHAEE enhanced fatty acid $\beta$-oxidation in peroxisomes and apparent DHA absorption and increased the liver DHA contents in rats. Therefore, when the same quantity of DHA was consumed, H-DHAEE consumption was more efficacious in improving lipid metabolism compared with L-DHAEE consumption. This study contributes to the development of supplements with the EE-form of DHA by clarifying the bioavailability of different degrees of DHA-EE.

\section{Acknowledgments}

We would like to thank Eiko Kimura, Hiroe Shimizu, Hiromitsu Imaike, and Midori Fukao of Kansai University, for their help with animal care and lipid analyses.

\section{References}

Berge, R. K., Madsen, L., Vaagenes, H., Tronstad, K. J., Göttlicher, M., \& Rustan, A. C. (1999). In contrast with docosahexaenoic acid, eicosapentaenoic acid and hypolipidaemic derivatives decrease hepatic synthesis and secretion of triacylglycerol by decreased diacylglycerol acyltransferase activity and stimulation of fatty acid oxidation. Biochemical Journal, 343(1), 191-197. http://dx.doi.org/10.1042/0264-6021:3430191

Bligh, E. G., \& Dyer, W. J. (1959). A rapid method of total lipid extraction and purification. Canadian journal of biochemistry and physiology, 37(8), 911-917. http://dx.doi.org/10.1139/o59-099

Bradley, R. L., Fisher, F. F., \& Maratos-Flier, E. (2008). Dietary fatty acids differentially regulate production of TNF-alpha and IL-10 by murine 3T3-L1 adipocytes. Obesity, 16(5), 938-944. http://dx.doi.org/10.1038/oby.2008.39

Frøyland, L., Vaagenes, H., Asiedu, D. K., Garras, A., Lie, O., \& Berge, R. K. (1996). Chronic administration of eicosapentaenoic acid and docosahexaenoic acid as ethyl esters reduced plasma cholesterol and changed the fatty acid composition in rat blood and organs. Lipids, 31(2), 169-178. http://dx.doi.org/10.1007/BF02522617

Guo, W., Wong, S., Xie, W., Lei, T., \& Luo, Z. (2007). Palmitate modulates intracellular signaling, induces endoplasmic reticulum stress, and causes apoptosis in mouse 3T3-L1 and rat primary preadipocytes. American Journal of Physiology - Endocrinology and Metabolism, 293(2), E576-E586. http://dx.doi.org/10.1152/ajpendo.00523.2006

Horton, J. D., Bashmakov, Y., Shimomura, I., \& Shimano, H. (1998). Regulation of sterol regulatory element binding proteins in livers of fasted and refed mice. Proceedings of the National Academy of Sciences, 95(11), 5987-5992. http://dx.doi.org/10.1073/pnas.95.11.5987

Hosomi, R., Fukunaga, K., Arai, H., Kanda, S., Nishiyama, T., \& Yoshida, M. (2010). Effect of dietary protamine on lipid metabolism in rats. Nutrition Research and Practice, 4(6), 462-469. http://dx.doi.org/10.4162/nrp.2010.4.6.462

Hosomi, R., Yoshida, M., \& Fukunaga, K. (2012). Seafood consumption and components for health. Global Journal of Health Science, 4(3), 72-86. http://dx.doi.org/10.5539/gjhs.v4n3p72

Ide, T., Watanabe, M., Sugano, M., \& Yamamoto, I. (1987). Activities of liver mitochondrial and peroxisomal fatty acid oxidation enzymes in rats fed trans fat. Lipids, 22(1), 6-10. http://dx.doi.org/10.1007/BF02534867

Ikeda, I., Imasato, Y., Nagao, H., Sasaki, E., Sugano, M., Imaizumi, K., \& Yazawa, K. (1993). Lymphatic transport of eicosapentaenoic and docosahexaenoic acids as triglyceride, ethyl ester and free acid, and their effect on cholesterol transport in rats. Life Science, 52(16), 1371-1379. http://dx.doi.org/10.1016/0024-3205(93)90172-Y

Ikeda, I., Sasaki, E., Yasunami, H., Nomiyama, S., Nakayama, M., Sugano, M., ... Yazawa, K. (1995). Digestion and lymphatic transport of eicosapentaenoic and docosahexaenoic acids given in form of triacylglycerol, free acid and ethyl ester in rats. Biochimica et Biophysica Acta, 1259(3), 297-304. http://dx.doi.org/10.1016/0005-2760(95)00180-8

Kelley, D. S., Nelson, G. J., \& Hunt, J. E. (1986). Effect of prior nutritional status on the activity of lipogenic 
enzymes in primary monolayer cultures of rat hepatocytes. Biochemical Journal, 235(1), 87-90.

Kelley, D. S., \& Kletzien, R. F. (1984). Ethanol modulation of the hormonal and nutritional regulation of glucose 6-phosphate dehydrogenase activity in primary cultures of rat hepatocytes. Biochemical Journal, 217(2), 543-549.

Kien, C. L., Bunn, J. Y., \& Ugrasbul, F. (2005) Increasing dietary palmitic acid decreases fat oxidation and daily energy expenditure. The American Journal of Clinical Nutrition, 82(2), 320-326.

Kris-Etherton, P. M., Harris, W. S., \& Appel, L. J., American Heart Association. (2002). Nutrition Committee. Fish consumption, fish oil, omega-3 fatty acids, and cardiovascular disease. Circulation., 106(21), 2747-2457. http://dx.doi.org/10.1161/01.CIR.0000038493.65177.94

Kris-Etherton, P. M., Harris, W. S., \& Appel, L. J., Nutrition Committee. (2003). Fish consumption, fish oil, omega-3 fatty acids, and cardiovascular disease. Arteriosclerosis, Thrombosis, and Vascular Biology, 23(2), e20-30. http://dx.doi.org/ 10.1161/01.ATV.0000038493.65177.94

Krokan, H. E., Bjerve, K. S., \& Mørk, E. (1993). The enteral bioavailability of eicosapentaenoic acid and docosahexaenoic acid is as good from ethyl esters as from glyceryl esters in spite of lower hydrolytic rates by pancreatic lipase in vitro. Biochimica et Biophysica Acta, 1168(1), 59-67. http://dx.doi.org/10.1016/0005-2760(93)90266-C

Lawson, L. D., \& Hughes, B. G. (1988). Absorption of eicosapentaenoic acid and docosahexaenoic acid from fish oil triacylglycerols or fish oil ethyl esters co-ingested with a high-fat meal. Biochemical and $\begin{array}{llll}\text { Biophysical Research } & \text { Communications, } & \text { 156(2), }\end{array}$ http://dx.doi.org/10.1016/S0006-291X(88)80937-9

Lefils, J., Géloën, A., Vidal, H., Lagarde, M., \& Bernoud-Hubac, N. (2010). Dietary DHA: time course of tissue uptake and effects on cytokine secretion in mice. British Journal of Nutrition, 104(9), 1304-1312. http://dx.doi.org/10.1017/S0007114510002102

Lowry, O. H., Rosebrough, N. J., Farr, A. L., \& Randall, R. J. (1951). Protein measurement with the Folin phenol reagent. The Journal of Biological Chemistry, 193(1), 265-275.

Maeda, K., Okubo, K., Shimomura, I., Mizuno, K., Matsuzawa, Y., \& Matsubara, K. (1997). Analysis of an expression profile of genes in the human adipose tissue. Gene., 190(2), $227-235$. http://dx.doi.org/10.1016/S0378-1119(96)00730-5

Markwell, M. A., McGroarty, E. J., Bieber, L. L., \& Tolbert, N. E. (1973). The subcellular distribution of carnitine acyltransferases in mammalian liver and kidney. A new peroxisomal enzyme. The Journal of Biological Chemistry, 248(10), 3426-3432.

Matsuzawa, Y. (2006). Therapy insight: adipocytokines in metabolic syndrome and related cardiovascular disease. Nature Clinical Practice Cardiovascular Medicine, 3(1), $35-42$. http://dx.doi.org/10.1038/ncpcardio0380

Moreno-Aliaga, M. J., Lorente-Cebrián, S., \& Martínez, J. A. (2010). Regulation of adipokine secretion by n-3 fatty acids. Proceedings of the Nutrition Society, 69(3), 324-332. http://dx.doi.org/10.1017/S0029665110001801

Nelson, G. J., \& Ackman, R. G. (1988). Absorption and transport of fat in mammals with emphasis on n-3 polyunsaturated fatty acids. Lipids, 23(11), 1005-1014. http://dx.doi.org/10.1007/BF02535644

Prevot, A. F., \& Mordret, F. X. (1976). Utilisation des colonnes capillaries de verre pour l'analyse des corps gras par chromotographie en phase gazeuse. Revue Francaise des Corps Gras, 23, 409-423.

Reicks, M., Hoadley, J., Satchithanandam, S., \& Morehouse, K. M. (1990). Recovery of fish oil-derived fatty acids in lymph of thoracic duct-cannulated Wistar rats. Lipids, 25(1), 6-10. http://dx.doi.org/10.1007/BF02562420

Rouser, G., Fkeischer, S., \& Yamamoto, A. (1970). Two dimensional then layer chromatographic separation of polar lipids and determination of phospholipids by phosphorus analysis of spots. Lipids, 5(5), 494-496. http://dx.doi.org/10.1007/BF02531316

Tanabe, T., Nakanishi, S., Hashimoto, T., Ogiwara, H., Nikawa, J., \& Numa, S. (1981). Lipids Part C. Methods Enzymol, Acetyl-CoA carboxylase from rat liver: EC 6.4.1.2 Acetyl-CoA: carbon-dioxide ligase (ADP-forming). New York, NY: Academic Press. http://dx.doi.org/10.1016/0076-6879(81)71003-6 
Tang, X., Li, Z. J., Xu, J., Xue, Y., Li, J. Z., Wang, J. F., \& Wang, Y. M. (2012). Short term effects of different omega-3 fatty acid formulation on lipid metabolism in mice fed high or low fat diet. Lipids in Health and Disease, 11, 70. http://dx.doi.org/10.1186/1476-511X-11-70

\section{Copyrights}

Copyright for this article is retained by the author(s), with first publication rights granted to the journal.

This is an open-access article distributed under the terms and conditions of the Creative Commons Attribution license (http://creativecommons.org/licenses/by/3.0/). 